\title{
A New Class of Low Cost, High Performance Radiation Detectors
}

\author{
Peter S. Friedman, Member, IEEE
}

\begin{abstract}
A new class of radiation detectors has been conceived and demonstrated using plasma panel TV-set technology for direct conversion of ionizing radiation to freeelectrons and a gaseous media for electron amplification with a gain of approximately $10^{11}$. For the basic configuration, a native pixel resolution of $10 \mu \mathrm{m}$ appears feasible, with a pixel response time of $1 \mu$ s or faster, and a manufacturing cost of less than \$1 per square inch for detector and electronics. New structures under consideration suggest the possibility that the plasma panel detector could be a transformational technology with low cost, large area, high spatial and temporal resolution, and potentially high efficiency, high energy spectral resolution and high neutron/gamma-ray discrimination.
\end{abstract}

\section{INTRODUCTION}

$\mathrm{T}_{\mathrm{s}, \mathrm{sec}}$ HE feasibility of a newly conceived [1] low-cost, hybrid solid state, ionizing radiation detector with high performance capability has recently been demonstrated [2]. This device, which constitutes a new class of radiation detectors, utilizes a gaseous media for electron amplification and can best be described as a plasma panel sensor ("PPS"). The PPS was conceived to take advantage of the existing technology base and manufacturing infrastructure for plasma display panel ("PDP") modules, which are being mass produced as large area (i.e., 1 to 2 meter diagonal), high definition, flat panel TV-sets and currently sell wholesale for about \$0.90 per square inch (including electronics).

\section{The Plasma Panel as a Radiation SEnsor}

The PPS is based on a simplified, open-cell, monochrome plasma panel structure (see Fig. 1). The first successful PPS devices tested were in fact PDP's that had originally been manufactured as alphanumeric, plasma display panels, and were never intended for use as radiation detectors. In spite of this, these test panels were successfully modified to function as radiation sensors, and demonstrated reasonable sensitivity to several gamma-ray sources, including ${ }^{57} \mathrm{Co}(122 \mathrm{keV}),{ }^{99} \mathrm{Tc}$ $(143 \mathrm{keV})$ and ${ }^{137} \mathrm{Cs}(662 \mathrm{keV})$. Although a variety of devices (i.e. AC- and DC-PDPs) have been successfully demonstrated, the best result was for a PDP with an active area of only 2.7 square inches, which was able to detect a $96 \mu \mathrm{Ci}$ source of ${ }^{57} \mathrm{Co}$, at a distance of 67 inches, corresponding to a radiation level at the panel's surface of $\mathbf{3 . 0} \boldsymbol{\mu r e m} / \mathbf{h r}$. For this device about $88 \%$ of the incident gamma radiation is estimated to have passed through the PDP without interaction. However

This work was supported in part by the U.S. Defense Threat Reduction Agency under Contract No. DTRA01-03-C-0042. from a materials design viewpoint, the above results are particularly impressive because of the non-optimum device structure. If this device had been designed from the onset to be a radiation detector (i.e. PPS) instead of an alphanumeric display panel (i.e. PDP), utilizing a proper conversion layer, and employing low electron affinity materials combined with higher fields for enhanced efficiency and discharge localization, an improved Penning gas mixture, and elimination of wall charge, a sensitivity and performance improvement of at least an order-of-magnitude improvement would probably have been achieved. As it turns out, in order for a PPS to function effectively as a radiation sensor, it must be structurally and electronically designed with essentially an opposite set of design rules as those governing its use as a display device.

Functionally, the PPS operates as a direct, high-gain, position and intensity sensitive, digital counter of electrons emitted as a consequence of the interaction of ionizing photons and/or particles with materials that internally convert such radiation into "free-electrons", without going through a scintillation conversion step and subsequent detection by an expensive photosensing element or requiring use of highpressure, high-purity isotopic gases such as ${ }^{3} \mathrm{He}$ or ${ }^{10} \mathrm{BF}_{3}$. To maximize performance, a high efficiency radiation "absorbing" conversion layer (or substrate) is incorporated into the basic plasma panel structure (see Fig. 1). With this addition, the PPS becomes a macro-array of parallel pixelsensor-elements capable of both detecting and imaging single free-electrons (without use of photomultipliers or semiconductors) generated by incident ionizing radiation. Typically such electrons escaping the panel wall into the gas will undergo electron multiplication within about 100 nanoseconds, resulting in a self-contained "plasma" gas discharge (i.e. avalanche) confined to the pixel cell space. For all plasma panel devices, this process is designed to be selflimiting by virtue of an opposing impedance in series with the pixel that cuts off the discharge by dropping the pixel voltage before too high a current develops. The output pulse from each pixel will therefore always be approximately the same, even if another "free-electron" enters the same local pixel space at the same moment in time. However, free-electrons entering a different pixel field space, even at the same time, will create a different discharge and be counted separately. This is the reason that plasma panels can act as intensitysensitive, digital-counting devices. In essence, given the very 
high spatial resolution capability (i.e. $\sim 10 \mu \mathrm{m}$ ) of the plasma panel electrode matrix, and the fast discharge response time, it is unlikely that two separate radiation generated freeelectrons will enter the same PPS pixel space at the same time. For these reasons, PPS devices can be intensity sensitive to radiation in a somewhat analogous manner as proportional counters, but with potentially much higher spatial resolution and speed than most scintillation-based radiation detectors. Thus, very high resolution images of radioactive emitters could be captured from, or of, a moving object in real time, be it a car or airplane, or a beating heart.

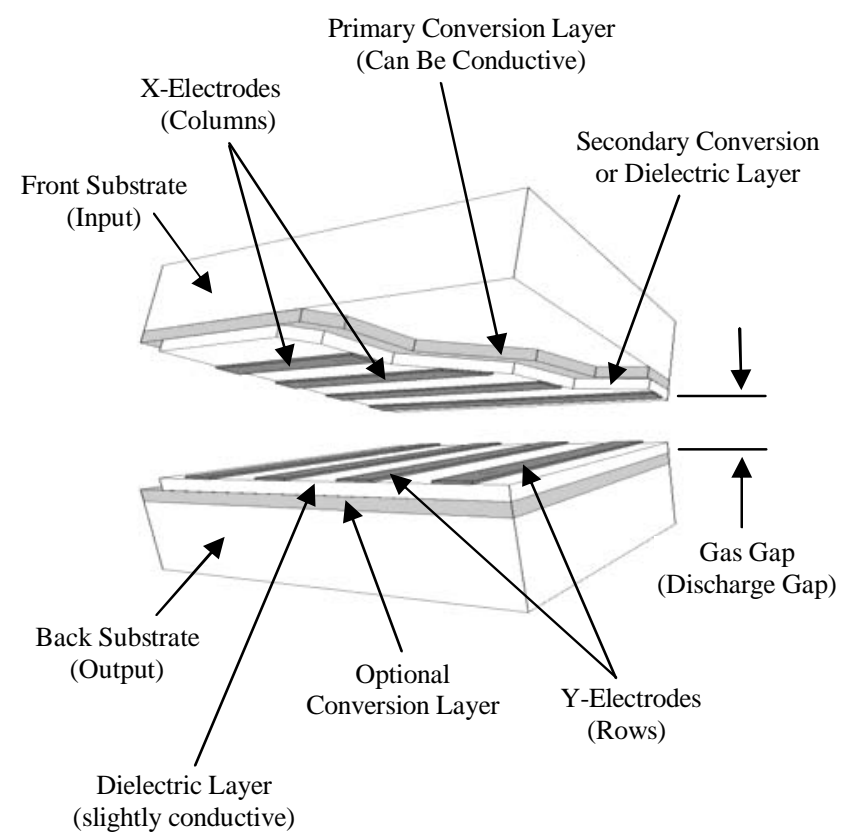

Fig. 1. PPS Front and Rear Plate Structure (with fill-gas between plates).

Unlike detection devices which tend to operate in the "Proportional Region" of the gas ionization curve with a typical gas multiplication gain of about $10^{4}$, the PPS operates at the top-end of the Geiger-Müeller region with an internal gain on the order of $10^{11}$, hence does not require signal amplification electronics. This is primarily due to the high pixel field strength of about $6 \times 10^{6} \mathrm{~V} / \mathrm{m}$, resulting from a combination of high PPS operating voltage ( $\sim 600$ volts) and very small anode-to-cathode gas gap of $\sim 100 \mu \mathrm{m}$. From the above description it should be clear that the PPS is a true digital integrating device. The discharge current of an individually lit pixel is unimportant, only the fact that a pixel is either "on" or "off" (i.e. lit or unlit). The intensity integration is therefore based on the number of times that a particular pixel turns "on" in a fixed period. With the possibility of a pixel lighting as many as a million times per second (i.e., $1 \mathrm{MHz}$ ) the response speed $(1 \mu \mathrm{s})$ or image gray scale capability of a PPS detector is most impressive and far better than for a PDP video monitor operating at a sustain frequency of $50 \mathrm{kHz}$ and able to display 256 shades of gray in 1/60 of a second. Besides pixel discharge data, the PPS electronics can capture the location of each pixel that goes "on". It is noted that for PPS devices, pixel uniformity should be even better than in a PDP, because the internal plasma panel structure is planar (see Fig. 1) as opposed to 3-dimensional (i.e. PDP devices require hard-to-control, 3-D process fabrication steps such as sintering and sandblasting of vertical barrier walls, etc.).

Given the above pixel response rate of $10^{6} \mathrm{cps}$, with a pixel pitch of $10 \mu \mathrm{m}$ corresponding to an image cell density of $10^{6}$ pixels $/ \mathrm{cm}^{2}$, yields a PPS signal response capability of $10^{12} \mathrm{cps}$ per $\mathrm{cm}^{2}$. This value is probably far in excess of anything that might be encountered, so signal saturation should not be an issue and PPS devices should be capable of providing a linear digital intensity response to almost any source of incident radiation. In terms of spectral range, the PPS sensitivity should be able to extend from about $20 \mathrm{keV}$ to well into the $\mathrm{MeV}$ (or possibly even the $\mathrm{GeV}$ ) range.

For applications involving a mixture of radioactive sources (e.g. homeland security), it is desirable that a radiation detector be able to respond with high sensitivity over a broad spectral range. Such a situation, however, leads to a conflicting choice of device materials, dimensions and optimization solutions. To address this multifaceted need, a multi-level device structure has been conceived that can exploit both the photoelectric and Compton effects via a micro-segmented internal PPS configuration. For example, at low energies the substrate plate and conversion layer thickness should be minimal in order to maximize incident radiation through the substrate and into the conversion layer, and then subsequent transport of resulting electrons through the conversion layer to the gas. In contrast, at high energies the substrate thickness is not a problem, as the primary issue is to achieve sufficient Compton scattering of incident radiation with the conversion layer for efficient generation of Compton recoil electrons and subsequent electron transport through the conversion layer into the gas. By combining a multi-level device structure with a modular component approach, an overall system design based on a tiled-array configuration can be implemented to achieve optimum system sensitivity over the broadest possible spectral range. An important benefit of the multi-level PPS configuration is that it can also be used to advantage for radioisotope source identification. In its most basic embodiment, the multi-level PPS can be configured as a dual-level, micro-segmented PPS detector (see Fig. 2), which can be viewed as a special case of the basic PPS configuration shown in Fig. 1. For example, a dual-level plateau metallic conversion plate could be thought of as simply a "patterned" metallic front substrate combined with a conductive conversion layer. The preferred material for such a conversion plate for X-ray/gamma-ray radiation would most likely be a high- $\mathrm{Z}$ metal that could be conveniently cast or otherwise formed into the chosen dual-level plateau (i.e. 
ribbed) pattern. It is noted that the front substrate does not have to be a metal; it could also be a patterned ceramic or glass incorporating high-Z materials with different plateau thicknesses for different optimization solutions over a broad range of incident photon energies.

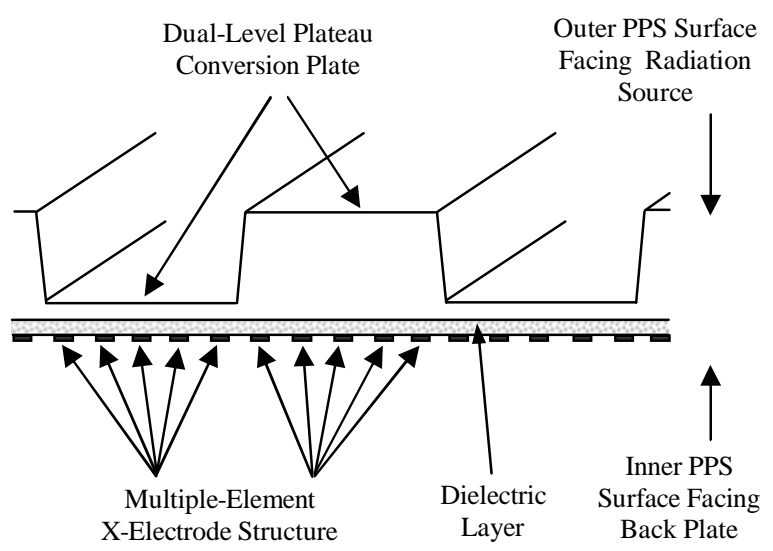

Fig. 2. Front Substrate of Dual-Level, Multi-Electrode, Micro-Segmented PPS.

The dual-level, micro-segmented, PPS configuration shown in Fig. 2, has been designed to take advantage of the inherent high spatial resolution resulting from the internal device micro-discharge cell structure. A trapezoidal cross-sectional shape is probably the most convenient for the dual-height plateau structure making up the outer, top surface of the conversion plate. The discharge electrodes located on top of the flat inner dielectric surface would be parallel to, and electrically isolated from, the outer rib structure, and would be by design, centered alternately under either the thin or thick plateau layers of the conversion plate. For a given photon flux incident upon the ribbed plate, a series of uniquely defined gas discharge intensity patterns and ratio differences should be observed between electrodes centered under a thin versus a thick conversion plate plateau.

In terms of the direct implications of the modified-PDP sensor devices already demonstrated and previously discussed, the above results could be restated as follows. A modified-PDP detected weak radioisotope emission (i.e, 3.0 $\mu \mathrm{rem} / \mathrm{hr}$ ) at a distance of 5 feet, or equivalently at 10 feet if two such 2.7 square inch detectors were located on opposite sides of a 10 foot portal opening. In other words, for cargo or vehicles moving through a 10 foot wide doorway, two small (i.e., 1 to 2 inch diagonal) PPS detectors could in principle detect emitted gamma-rays at or below the background radiation level. It cannot be over-emphasized that this data comes from a PDP device originally designed to be a display panel and not a radiation detector. Further, because the technology is so inexpensive, a passive portal based on only a few square inches of PPS detector does not make much sense. Instead, one should use at least a few square meters of PPS detector to capture higher quality source images, at fast speeds (e.g. $60 \mathrm{mph}$ ), and with sufficient spectroscopic resolution for radioisotope identification.

At the current stage of development (that is without an adequate experimental database or suitable theoretical model), it is too early to quantify PPS device efficiency other than to say that there are no obvious limitations to the technology, and that a Monte Carlo based modeling program to calculate device efficiencies is under consideration. However, for many radiation detector applications the intrinsic device efficiency is not the critical parameter. The most important parameter is the global or system efficiency. This is because the only way to beat global $1 / \mathrm{r}^{2}$ efficiency losses, is for the detector system to "cover" the largest possible surface area, so as to collect the largest solid angle of emitted radiation; however, this can only be practically achieved if the detector is very low in cost. The projected PPS device cost on the order of $\$ 1$ per square inch is approximately one to two, orders-of-magnitude less expensive than competing "conventional" systems, and so can be taken maximum advantage of by employing large detector areas to achieve correspondingly high system efficiencies.

\section{PPS RADIATION RESPONSE}

For gas-filled radiation counters, significant reaction to low-energy gamma-ray/X-ray or thermal-neutron radiation can occur both in the gas detection media and the device walls. However, even for device fill-gases with the highest attenuation cross-sections (e.g. Xe for gamma-rays and ${ }^{3} \mathrm{He}$ for slow-neutrons), very little interaction can occur in the gas if the device operates at a reduced pressure and has a small gas gap, as is the case for the PPS. With higher-energy radiation, the "absorption" will occur almost exclusively in the device walls, assuming the walls are made of appropriately high attenuation materials. Yet only electrons that manage to escape from the wall surface into the gas can be amplified and counted. Thus the fill-gas in the PPS does not functionally serve as the conversion media, but as the signal amplification media, and for this reason the PPS should not be thought of as a gas detector, but rather as a hybrid solid state detector.

In contrast to ionizing photon detection, the response of a PPS device to slow-neutrons can be either via a single-step or multi-step reaction process, including direct emission of an internal conversion electron by a suitable conversion layer material, such as ${ }^{157} \mathrm{Gd}$. Incident slow-neutrons can therefore directly or indirectly (i.e. via gamma-ray emission) generate free-electrons that can be counted/imaged by the PPS. The PPS can also be designed as a fast-neutron detector by modification of the conversion layer structure, device materials and dimensions. As with conventionally designed neutron detectors, fast-neutrons are more difficult to detect and most often involve incorporating materials containing ${ }^{1} \mathrm{H}$ and/or ${ }^{6} \mathrm{Li}$, however, the basic PPS device advantages would 
still be retained (it is noted that the PPS structure allows for the use of interesting material possibilities, such as $\mathrm{LiH}$, that have the potential to significantly improve fast-neutron efficiency). Finally the PPS should also be capable of detecting a variety of other ionizing species including charged particles, whether generated internally as a result of high energy incident photons or neutrons, or externally by an incident source.

Any device that responds to incident radiation by generating a number of information carriers in proportion to the energy of the radiation, can in principle, be configured to yield a representative spectral distribution energy curve of the incident radiation for radioisotope identification purposes. Since low bandgap semiconductors, such as germanium, produce approximately an order-of-magnitude more information carriers (i.e. electron-hole pairs) than higher bandgap scintillator-based devices (i.e. photons and photoelectrons), semiconductor-based detectors should be, and are, capable of realizing significantly higher energy resolution than scintillation detectors. For the PPS device, the number of information carriers (i.e. free-electrons) generated per radiation interaction event is not expected to be large relative to that of a semiconductor- or scintillator-based device. Therefore on a strictly equal comparative basis, the "intrinsic" energy spectral resolution for the basic PPS structure as shown in Fig. 1 would not be expected to be high. However, the distinctive properties of the PPS have led to the conception of new PPS configurations [3] that should enable important system-level enhancements which could greatly improve the PPS system energy spectral resolution, as well as device efficiency and temporal response, while also providing high neutron/gamma-ray discrimination for detecting and identifying radioisotopes within a complex mixture of source radioactive materials (e.g. for homeland security applications). Finally it is noted that spectral resolution generally improves with detector size, because FWHM is approximately inversely proportional to the square root of the number of counts. In this regard, the PPS low cost can lead to the use of large detector areas to increase the number of counts and thereby improve spectral resolution and radionuclide identification.

In general, the greater the energy of ionizing radiation incident upon a detector, the larger the number of downstream electron scattering events occurring in close proximity to the original interaction site. The resulting scattering pattern or "cluster" of secondary electrons should thus be characteristic of the incident radiation spectral distribution, and the higher the pixel resolution of the detector, the greater the cluster pattern detail (i.e. information). By employing various conversion layer thicknesses (corresponding to different spectral sensitivity PPS devices), in a tiled PPS module array detection system, a corresponding matrix of equations can be generated that in principle could be used to deconvolute the incident energy spectrum via a spectrum unfolding process [4], thereby providing radioisotope spectroscopic identification information. However, for the PPS configurations shown in Figs. 1 and 2, the resulting spectral energy resolution achievable via the above described spectrum unfolding process is not expected to be particularly impressive (e.g. relative to crystalline scintillation detection devices). The ability to attain truly high energy spectral resolution for PPS devices will therefore depend on new PPS configurations [3].

Any optimization of the PPS device will be largely dependent upon optimization of the conversion plate/layer, which will always involve some level of compromise. For example, the thicker the conversion plate/layer, the greater the radiation absorption, but the smaller the probability will be of generated free-electrons actually reaching the gas. Optimization thus involves a balance between absorption and electron transport, because these two functions move in opposite directions. The efficiency bar or balance point however, can be raised by vertically stacking one PPS on top of another, which provides the system designer with a new degree of freedom. Use of such a configuration, will shift the optimization balance towards individually thinner conversion layers (i.e. less absorption), with each plate/layer having improved electron transport, and making up for the reduced absorption by vertically stacking more devices on top of each other. This solution is readily affordable since each PPS device is individually low in cost. The extra degree of freedom associated with vertical stacking also allows for a variety of structural combinations, such as: having different spectral response optimized devices on top of each other, or different dual-level plateau structures on top of each other, or devices with different materials sandwiched together (e.g. different gas mixtures, different conversion layers, etc.). In fact, the vertical stack can even be monolithic, with the back substrate of the first device, serving as the front substrate of the second device, and so on, etc. It is noted that the monolithic stacked concept has been successfully employed in other fields to improve device efficiency, such as for compound, multi-bandgap photovoltaic devices. In summary, the benefits of PPS vertical stacking are three-fold: (1) improved device efficiency, (2) improved spectroscopic resolution, and (3) improved source angular/directional resolution. However, important additional advantages can potentially be realized from a vertically stacked device structure by eliminating the use of "conventional" image collimators which result in detectors being "photon starved." This can be accomplished probably most directly through the use of Compton imaging techniques [5], which should be able to provide the angular component (or information) required for radioactive source imaging, while also enhancing signal strength, and reducing device weight, bulk and cost. 
In terms of additional advantages, the PPS requires neither high vacuum nor high purity gases, and thus no getters are needed. Instead of a costly and possibly fragile scintillation plate, the PPS can employ relatively inexpensive "conversion layers." Also there is no need for expensive photodiodes or photomultiplier tubes or fiber optic coupling, since electronic detection in the PPS is by directly monitoring the pixel discharge across the small gap separating the two substrate plates. In terms of cost, the PPS does not employ signal amplification electronics or require cryogenic cooling schemes for noise reduction. In summary, the most obvious advantage of the PPS technology lies in its tremendous cost reduction potential of one to two, orders-of-magnitude. Yet perhaps equally important for many applications are the potential spatial and temporal resolution advantages. In particular, with a pixel pitch on the order of $0.01 \mathrm{~mm}$, the PPS has the capability of providing one to two, orders-ofmagnitude better device resolution than the current generation of radiation imagers.

\section{ORDER-OF-MAGNITUdE SENSITIVITY IMPROVEMENT: THE ROLE OF PRIMING ElECTRONS}

In order for a plasma panel to function as a radiation sensor, it must be configured such that the "priming" electrons (needed to initiate the high-gain discharge) come from an external source of ionizing radiation (e.g. gammarays, neutrons, etc.). This essentially means that the plasma panel must be structurally and electronically designed with an opposite set of design rules as those governing its use as a display device. Thus, to maximize sensitivity, the panel must be designed to eliminate or minimize all possible internal sources of priming electrons. Unfortunately, all of the PDP sensors used in the DTRA demonstration program [2] were originally built to function as display devices, meaning that these plasma panels were all designed to have good internal priming. As a result, the problem of charge storage and internal priming during the initial experimental effort was never fully overcome. However this problematic situation will not exist for future plasma panels designed from inception to function as radiation sensors (i.e. PPS's, not PDP's). In the discussion below, the PPS design elements and methodology for realizing order-of-magnitude performance enhancements are discussed in detail.

\section{A. Gas System and Priming Electrons}

The gas mixture, discharge gap, pressure and drive voltages, in combination need to inhibit all internal sources of priming electrons, while at the same time maximizing sensitivity to radiation-induced, free-electrons. Priming electrons can result in spurious pixel discharges (i.e. noise), but more importantly can lead to out-of-control discharge spreading. The method chosen below and previously demonstrated [2] to inhibit unwanted priming, should also minimize the number of gas-phase metastables, as well as the lifetimes of gaseous excited state species and propagation of VUV emitted photons via the use of appropriate gas-phase quenching and VUV absorbing molecules.

1) Penning Mixture - Addition of suitable, low ionization potential gaseous component(s) at low concentration to depopulate excited state metastable species. The Penning mixture maintains high amplification and hence good avalanche initiation.

2) Discharge Spreading Inhibitor - Elimination of adjacent cell priming to maintain spatial integrity of the initial localized discharge site, preventing the discharge from spreading into neighboring cells. The method successfully demonstrated involved adding a small amount of gas with a large electron capture cross-section and moderate electron affinity, thus reducing the mean free path of high mobility gaseous electrons and ions. It was spectroscopically demonstrated that $\mathrm{O}_{2}$ is such a gas and appears to form a Penning mixture with $\mathrm{Xe}$, thereby depopulating excited state metastable species. In addition, $\mathrm{O}_{2}$ will absorb VUV photons thus further localizing the pixel discharge. In essence, the addition of $\mathrm{O}_{2}$ demonstrated that the discharge can be controlled and suppressed enough to prevent it from spreading to adjacent cells, but not so much as to prevent localized (i.e. single pixel) avalanche amplification.

3) Gas Collisional Cross-Section and Discharge Voltage Panel gas mixtures at maximum pressure and with maximum average atomic-weight can be used to increase the likelihood of electron interaction and the probability of secondary electron generation. For example, the basic Xe in Ne mixture used in PDPs was modified to increase electron collisional cross-sections by replacing $\mathrm{Ne}$ with higher atomic-weight gaseous components. As a side benefit, increasing average atomic-weight can: (1) raise voltage thus increasing the local pixel field, (2) shorten discharge time to allow faster response and higher update rates, and (3) reduce device dead-time.

\section{B. Oxygen as a "Penning" Charge-Transfer Dopant and Avalanche Control Agent}

The visible emission spectra from two lighted AC-PDPs (i.e. undergoing discharge), filled with $100 \% \mathrm{Xe}$, and $99 \%$ $\mathrm{Xe} / 1 \% \mathrm{O}_{2}$, respectively, have been recorded. In the $100 \% \mathrm{Xe}$ panel, the well-known "blue" Xe-I emission was observed, with the two strongest visible lines readily apparent at 467 and $462 \mathrm{~nm}$. In the $99 \% \mathrm{Xe} / 1 \% \mathrm{O}_{2}$, even though the gas mixture still contained $99 \% \mathrm{Xe}$, the prominent "blue" Xe-I lines were "missing", but the strong "green" lines from 533 to $558 \mathrm{~nm}$ of ionized atomic oxygen were present and dominated the spectrum. The first ionization constants for molecular $\mathrm{O}_{2}$ and atomic $\mathrm{Xe}$ are respectively at 12.07 and $12.13 \mathrm{eV}$ (Ref.: NIST Ion Energetics Database). Thus molecular $\mathrm{O}_{2}$ and atomic $\mathrm{Xe}$ are coincidentally almost in perfect resonance, with the ionization energy of $\mathrm{O}_{2}$ being just below that of Xe, making $\mathrm{O}_{2}$ an excellent component for transferring the charge from ionized $\mathrm{Xe}$ to $\mathrm{O}_{2}$. The fact that 
the emission spectrum of the $99 \% \mathrm{Xe} / 1 \% \mathrm{O}_{2}$ gas mixture has the characteristic "green" color of oxygen, without any of the "blue" bands of xenon, shows that excited Xe efficiently transfers its energy to molecular $\mathrm{O}_{2}$, which upon dissociation (in an active plasma) emits from its lower energy levels centered around the $538 \mathrm{~nm}$ peak that characterizes atomic oxygen. From the spectroscopic data, it was therefore demonstrated that a small amount of $\mathrm{O}_{2}$ can effectively quench ionized $\mathrm{Xe}$ and excited Xe metastable states, and thereby confine the PPS discharge to the local pixel site closest to where the initial radiation "absorption" to freeelectron conversion event takes place. The fact that $\mathrm{O}_{2}$ is also a good VUV absorber and scavenger of "stray" electron charge simply adds to its effectiveness as both a discharge spreading inhibitor and avalanche control agent.

\section{Free-Electron Conversion Plate/Layer}

For efficient conversion of ionizing radiation to freeelectrons and subsequent electron transport to the gas, it is necessary to use materials having: (1) an appropriate thickness to match both the attenuation coefficient with respect to the incident radiation and the electron range with respect to the emitted free-electron, (2) good electron emissivity in the conversion media (i.e. high electron-fraction associated with photoelectron and Compton recoil electron emission), (3) efficient electron transport through the conversion media to the gas through use of low (or negative) electron affinity materials, (4) minimization of the number of interface layers to reduce electron traps caused by interface defects, and (5) a low surface work-function with regard to interface layer surfaces, especially for electron ejection out of the panel wall into the gas.

1) Front Substrate / Conversion Plate - In principle, PPS device efficiency can be enhanced by using materials that serve the combined role of front substrate and conversion plate. At the very least, such a configuration will reduce interface losses. Such materials can include metals, ceramics and/or glass. For conductive substrates, a weakly conducting "insulator" will need to be deposited on the substrate bottom surface for both electrode isolation and to prevent accumulation of stored wall charge. Most of the standard electron beam or sputtered thin-film oxides should work as a dielectric, assuming a reasonable match between the linear coefficients of thermal expansion; ideally such dielectrics such have a low work-function for secondary-electron emission. If necessary these materials could be made slightly conductive by the addition of $\mathrm{Li}_{2} \mathrm{O}$, as the $\mathrm{Li}^{+}$ion is known to be highly mobile and could therefore provide enough conductivity to bleed off the offending pixel wall charge. As an alternative to the above described metal conversion plate, a "conductive ceramic" nitride such as TaN could be used. Other nitrides of interest might include $\mathrm{HfN}$, TiN and BN. It is noted that all of the above ceramics are based on combining elements with either low or negative electron affinities. With respect to electrode materials, good candidates should have high electron emissivity and low electron affinity.

2) Conversion Plate Optimization - A primary factor in maximizing PPS device performance lies in optimization of the PPS conversion plate material and configurational structure. The choice of materials, configuration and dimensions is critical. Preliminary PPS designs can be evaluated by theoretical modeling and device simulation using Monte Carlo type numerical tools. The PPS modeling effort will need to take into account the configurational structure and device dimensions, material attenuation coefficients, material electron affinity, free-electron range, material emissivity, and surface work-function with regard to electron ejection into the gas.

\section{Panel Materials, Electron Affinity and Wall Charge}

Electron affinity (E.A.) measures the energy released upon the capture of an electron. High positive E.A. materials such as the halogens are therefore electron scavengers and to be avoided in the plasma panel structure. By implication, low E.A. materials such as iron, tantalum, titanium and boron are much better, and negative E.A. materials such as magnesium are especially desirable. All of the inert gases have negative E.A. values, hence are compatible with the build up of an electron gas discharge avalanche and so have been used in PDP's. Nitrogen and mercury also have negative E.A.'s, and so mercury-doped inert gas mixtures have been used for decades in DC-PDP's. With respect to electrodes, the alkaline-earth metals all have negative E.A.'s, along with some of the transition metals such as manganese, zinc and hafnium. Low cost, low numerical value E.A. materials such as $\mathrm{Hg}, \mathrm{Mn}$ and $\mathrm{Zn}$ are used in batteries, whereas most of the alkaline-earth metal oxides have been used as electron emitters in CRT's and as secondary electron emitters for photomultiplier dynodes. It follows that high E.A. materials such as the oxidized lead based thick film dielectric used in the PDP devices tested under the DTRA program need to be avoided in a properly designed PPS device. Based on the above considerations, reasonable material possibilities for the conversion plate might include: $\mathrm{Mn}$ alloyed with $\mathrm{Fe}$ in stainless steel (e.g., 18-18 PLUS, which is $\sim 18 \% \mathrm{Mn}$ ); Gd, and $\mathrm{Ni}$ alloyed $\mathrm{Gd}$; $\mathrm{Zn}$ alloyed $\mathrm{Cu}$ (e.g. brass can contain up to $\sim 45 \% \mathrm{Zn})$; Hf, and Hf alloyed with $\mathrm{Mn}$, Ta and/or Ti; and $\mathrm{Mg}$ and $\mathrm{Mg}$ alloys. It is noted that the first two alloys (i.e. the S.S. and Ni-Gd) are well matched to the linear expansion for PDP glass back-substrates and seal materials.

PPS devices need to be hermetically sealed, gas processed and generally fabricated in a manner similar to PDP devices. However, PDP's are designed to maximize wall charge (in part to enhance priming), which is exactly the opposite of what is desired for PPS devices. Since wall charge is the accumulated charge stored on the top internal surface at the panel gas interface, it is dynamic and therefore a source of priming electrons. To minimize wall charge, PPS devices 
need to avoid internal 3-dimensional dielectric surface structures, such as phosphor walls, barrier walls, isolation channels, dielectric spacers, phosphor particles, etc. Any 2dimensional dielectric surface in contact with the gas should have some level of conductivity to provide a mechanism to "bleed off" residual charge stored at the gas-wall interface.

\section{E. Spatial Resolution and Data Collection}

Depending upon electrode-pitch, the PPS is a highly integrated array of $10^{2}$ to $10^{6}$ micro-detection cells per square inch, each of which can act as an independent, position and intensity sensitive, radiation sensor. From a materials and fabrication viewpoint, a PPS pixel resolution of $\sim 0.01 \mathrm{~mm}$ is eminently feasible, much more so than for a PDP product. The reasoning behind this is fundamental and based on the fact that all of the PPS configurations under consideration are variations of $\boldsymbol{D C}$-PDP type structures, as opposed to the $\boldsymbol{A C}$ PDP structures found in virtually all commercial PDP-TV products. From a fabrication viewpoint, the electrode resolution in a DC-PDP can be much higher, and much better controlled, than in an AC-PDP device, because DC-PDP electrodes are not encapsulated under a highly reactive and chemically corrosive thick-film dielectric, which tends to undercut and undermine the AC-PDP electrode-material linewidth [6]. As an important collateral benefit, minimizing the PPS electrode width for purposes of enhancing image resolution, should also raise the intrinsic firing voltage, thereby increasing the local-pixel, electric-field strength, and hence the device sensitivity.

In terms of collecting the image location information (i.e. output data) of activated PPS pixels, each row and column of the PPS device needs to have a current limiting impedance (e.g. typically a resistor) to prevent run-away discharges, and so the location of each discharge event can be determined by measuring the row-column voltage drop. This method requires careful avalanche control (i.e. impedance matching) and fast response times to prevent data-loss due to PPS device dead-time. It is noted that instead of using an external row and column impedance, an internal impedance element (e.g. resistor) could be screen printed into each pixel to reduce the row/column discharge dead-time and so allow each pixel to operate independently of other pixels on the same electrode line.

\section{Electronic Circuitry, Signal Response and Control}

A pixel discharge or discharge event in a plasma panel is made self-limiting by virtue of an opposing impedance that cuts off the discharge before a run-away current develops. Thus whenever a discharge occurs, the current flowing through the electrodes and their associated impedances, causes a voltage drop across the impedances which terminates the discharge. The momentary voltage drop across the impedances in the current path of the discharging electrode can be sensed by a pulse detector. Proper design of the detection event circuitry involves optimization of the $\mathrm{X}$ and $\mathrm{Y}$ electrode circuit impedances, the DC (or AC) power level, the driver waveforms, the pulse detectors and almost every aspect of the panel's configuration and materials selection. It is noted that the electrode impedances may be implemented as either resistances and/or reactances and can involve either passive and/or active circuitry. In any case, the detection event circuitry should be designed not only to count the number of detection events, but to integrate the number of events per unit time and to record their spatial location in order to generate an image of the radiating source.

The PPS detector is expected to be a very low power consumption device, as it is based on a DC-PDP type structure which only consumes power upon pixel discharge/avalanche events and only in direct proportion to the number of avalanches. Under normal operating conditions (i.e. $\sim 99 \%$ of the time), the PPS would be detecting only minimal background radiation. Even under the worst case scenario of finding a powerful emitting radiation source, the PPS would not be expected to have nearly as many "lit" pixels as a PDP operating as a TV-video display. In fact, since the PPS operates in the DC mode, whereas all PDP television/video monitors operate in the AC mode, the PPS will not experience the significant parasitic losses of the capacitive AC sustain circuitry found in all PDP-TV appliances.

\section{A. Avalanche Control and Response Time}

An electron avalanche (i.e. plasma discharge) has features that need to be controlled: initiation, duration, reset and discharge spreading. Essentially the entire plasma panel material hierarchy and electronics reactive circuitry, contribute to the discharge avalanche characteristics. The specific control parameters include: gas mixture composition and pressure, discharge gap, electrode width and pitch, dielectric constant(s), dielectric cross-sectional thickness, dielectric surface and volume resistivity, discharge cell internal impedance, panel electrode row and column impedance, external reactive circuit impedance including power supply, etc. Since minimization of wall charge, along with spreading avalanches and pixel discharge resetting, were previously observed [2] to be problematic with AC-PDP's but not with DC-PDP's, the PPS configuration shown in Fig. 1 is based on DC-PDP type structure [7]. The two critical metrics required to meet the goals for avalanche control and fast response time are: (i) limit the discharge spreading to the spatial resolution of "one cell", and (ii) limit the cumulative time period of the three avalanche phases (i.e. initiation, duration and reset) to less than $1 \mu \mathrm{s}$, which was the approximate observed time period under the previous study (using $1.0 \mathrm{~mm}$ electrode pitch PDP devices). However, using a higher resolution panel (e.g. 0.01 electrode pitch), with a shorter discharge gap, and higher voltage, should lead to a 
significant reduction of the three avalanche phases to well below $1 \mu \mathrm{s}$. Yet even with a $1 \mu$ s pixel response time, each pixel should be capable of recording $10^{6} \mathrm{cps}$. Since the pixels in a PPS normally operate independently and in parallel, then a $10 \mu \mathrm{m}$ electrode pitch panel should be capable of recording $6 \times 10^{12} \mathrm{cps}$ per square inch.

\section{B. Buffer Circuit, Impedance, Data Processing and Noise}

As described above, in-line impedance is a factor in the initiation, duration and reset phases of an avalanche. Buffer circuitry can prevent feedback of an avalanche across one set of opposing electrodes from coupling into other sets of electrodes. A buffer circuit can incorporate in-line impedance and/or discharge-event sensing. It can also provide gating or synchronization of the power supply output to an electrode. The use of buffer circuits, with in-line impedance control, is highly interrelated with the power supply, gas mixture and electrode systems. These systems must integrally support the initial surge (initiation), and sustain it long enough (duration) to sense a discharge-event, but not so long as to enable discharge spreading, or electrode deterioration. The reset phase is for dissipation of gaseous excited state species, space/surface charges, and re-synchronized electronics. The initiation, duration and reset of the discharge-events under the previous study were observed to occur within $\sim 1 \mu \mathrm{s}$. It is noted that the power supply will need to be under control of the discharge-event signal processing system.

The outputs of the column electrode (X) and row electrode (Y) discharge event sensors are connected to synchronizing circuits that essentially time-stamp and reference each event to a master clock. X-Y synchronizing circuitry provides information to a "Sensitivity Control" circuit that in turn provides control to the power supply and the X-Y discharge event sensors. Thus, the discharge event processing system is a closely linked, if not closed loop, system that requires very coordinated integration with a variety of interrelated components. The discharge event processing system also feeds a stream of sequential electrode discharge event frames or "pictures" through a "Link" to a "Sensor Compiler." These frames or "pictures" represent the most basic imaging function of the PPS and can be compiled into segments in the time domain indicating intensity of radiation by mapping discharge event spatial and temporal density. Data and image processing can be enhanced by system level algorithms in the "Sensor Compiler" and "Detector Processor."

In terms of signal-to-noise, the projected $\mathrm{S} / \mathrm{N}$ ratio for the PPS should be high, as the plasma panel itself does not employ any low voltage, semiconductor structures such as transistors, or any other materials having significant temperature performance dependence. In fact, as discussed earlier, in terms of the number of information carriers generated, the PPS can be thought of an inherently high band gap device, which directly implies low thermal noise. Since there are no low band gap materials to be thermally populated, there is no reason to employ device cooling.
In fact, PDP devices, which are normally constructed using only inorganic amorphous type materials (e.g. glasses, ceramics, metals and gases) generally have the widest operating temperature range of any display device known and are essentially limited not by the plasma panel, but by the thermal range of the external electronic circuitry which is normally defined by the temperature range associated with solder joint integrity.

\section{CONCLUSION}

The feasibility of a new class of potentially very low cost, large area, high performance radiation sensors has been demonstrated for the detection of high energy photons (e.g. $\mathrm{X}$-rays/gamma-rays), neutral particles (e.g. slow and fast neutrons) and charged particles. A compelling attribute of this new class of ionizing radiation detectors is that the device conceptual design, structure, materials and fabrication process, heavily leverages off of the existing and substantial technology base and commercial manufacturing infrastructure already in place for high definition, plasma display panel (PDP) TV-sets. In actuality, the current $3^{\text {rd }}$ generation of high volume PDP production lines are probably too advanced to efficiently manufacture the PPS devices at anticipated volumes, however the older and most likely underutilized $1^{\text {st }}$ generation production lines are probably close to a perfect manufacturing fit for the fabrication of these devices.

Although the basic PPS structure and operational theory has been described in detail above, a number of more advanced embodiments of this technology [3] are under intensive evaluation and being considered for a multitude of applications. These applications cover everything from nuclear physics to homeland security, and from simple medical digital X-rays to order-of-magnitude advances in noninvasive imaging that could replace currently risky diagnostic surgical procedures. In summary, the new class of PPS radiation detector devices has the real potential of demonstrating the required combination of attributes necessary to constitute a transformational technology.

\section{REFERENCES}

[1] Patents pending U.S. and foreign. Assignee: Integrated Sensors, LLC, Toledo, Ohio 43606 (PH/FX: 419-536-3212; iSensors@ sbcglobal.net).

[2] Demonstration performed Oct. 2003 under Contract DTRA01-03-C-0042 to the U.S Defense Threat Reduction Agency.

[3] New patent applications currently being filed (disclosure of these configurations is planned for NSS/MIC 06).

[4] G. F. Knoll, Radiation Detection and Measurement, 3rd ed., John Wiley \& Sons, 2000, pp. 704-706.

[5] E. A. Wulf, B. F. Phlips, W. N. Johnson, J. D. Kurfess and E. I. Novikova, "Thick Silicon Strip Detector Compton Imager," IEEE Trans. Nucl. Sci., vol. 51, no. 5, pp. 1997-2003, Oct. 2004.

[6] P. S. Friedman, "Materials issues related to large-size color plasma displays," Invited Paper at $14^{\text {th }}$ International Display Research Conference (IDRC 94), Monterey, CA, pp. 112-117, Oct. 1994.

[7] DC-PDPs have been in commercial production since the 1970's. 